\title{
A case of inverted cystic tubulovillous adenoma of the duodenum
}

\author{
Hyung Ku Chon ${ }^{1}$, Hee Chan Yang ${ }^{2}$, Keum Ha Choi ${ }^{2}$, and Tae Hyeon Kim ${ }^{1}$
}

Departments of ${ }^{1}$ Internal Medicine and ${ }^{2}$ Pathology, Wonkwang University Hospital, Iksan, Korea

Received: September 13, 2018 Revised : November 14, 2018 Accepted: November 21, 2018

\section{Correspondence to}

Tae Hyeon Kim, M.D.

Tel: +82-63-859-2564

Fax: $+82-63-855-2025$

E-mail: kth@wku.ac.kr
A 67-year-old woman with a 3-month history of dyspepsia was referred to our department for the evaluation of an incidentally detected duodenal mass. She had no personal or social history, and she had been taking an anti-hypertensive medication for 10 years. On upper gastrointestinal endoscopy, an opened pomegranate-like shaped tumor measuring about $3.5 \mathrm{~cm}$ in diameter was observed in the second portion of the duodenum (Fig. 1A). The proximal part of the lesion showed a 1.5-cm bulge with smooth surface mucosa and an orifice oozing clear fluid at the top (Fig. 1B). Duodenoscopy revealed a papillary lesion with two central depressions and intact ampulla of Vater (Fig. $1 \mathrm{C}$ and $1 \mathrm{D}$ ). A biopsy specimen from the lesion revealed low-grade adenoma. Contrast-enhanced computed tomography showed an enhanced polypoid mass in the second portion of the duodenum (Fig. 2A). Endoscopic ultrasound demonstrated a heterogenous thick frondlike mass with cystic change arising from mucosa layer without abnormal lymph nodes, but submucosa intact and appears as an outer hyperechoic rim (Fig. 2B). Following the preoperative check-up, exploratory laparotomy was conducted and wide excision of the duodenal mass was successfully performed. Histology revealed cystic changes in the mucosa with tubulovillous growth along Brunner's glands into the deep submucosa, like an inverted shape (Fig. 2C). An inverted cystic tubulovillous adenoma involving Brunner's glands of the duodenum was confirmed. The patient was discharged without any complications, and follow-up endoscopy performed after 3 months showed a post-operative scar without a remnant tumor.
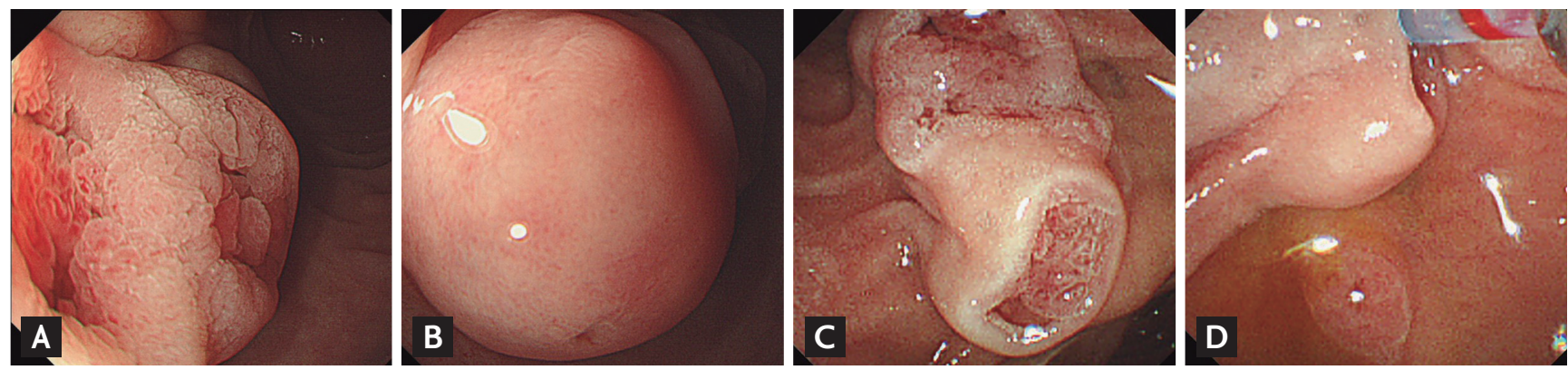

Figure 1. Endoscopic findings of the duodenal mass. (A) A tumor measuring about $3.5 \mathrm{~cm}$ in diameter, with an opened pomegranate-like shape, was noted in the second portion of the duodenum. (B) The proximal portion of the lesion showed hemispherical bulging mucosa with a smooth surface and an orifice releasing serous discharge. (C) On duodenoscopy, a longitudinal mass with two central depressive papillary lesions was observed with (D) intact ampulla of Vater. 

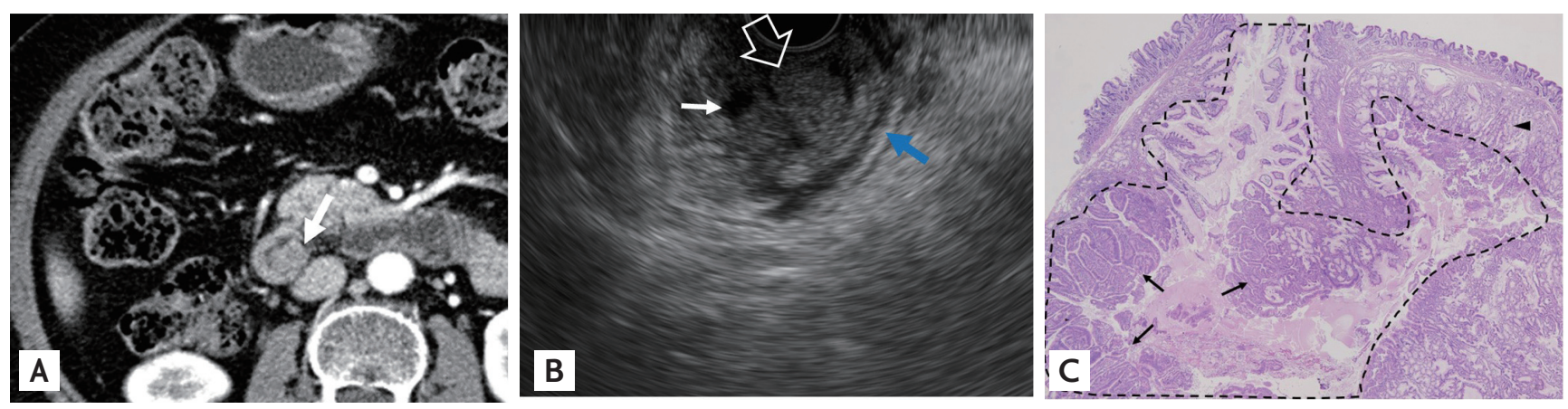

Figure 2. (A) Contrast-enhanced abdominal pelvic computed tomography showed a lobulated enhancing mass (white arrow) measuring $2.5 \mathrm{~cm}$ in the second portion of the duodenum. (B) Endoscopic ultrasound imaging revealed a heterogenous thick frondlike mass (open arrow) with cystic degeneration (white arrow) arising from mucosa layer, but submucosa intact and appears as an outer hyperechoic rim (blue arrow). (C) The tubulovillous adenoma (black arrows) involved Brunner's glands (black arrowhead) in the submucosa, cystically growing in an inverted pattern (H\&E, ×10).

Non-ampullary duodenal adenomas are infrequent observations, accounting for about $0.03 \%$ to $0.1 \%$ of diagnostic endoscopy findings. Furthermore, tubulovillous adenomas of the duodenum are extremely rare, and so far, there has been only one case reported previously of an inverted tubulovillous adenoma progressing to cystic degeneration involving Brunner's glands of the duodenum. We reported an unusual case of an inverted cystic tubulovillous adenoma involving Brunner's glands that was successfully diagnosed by surgical resection.

Written informed consents were obtained.

\section{Conflict of interest}

No potential conflict of interest relevant to this article was reported.

\section{Acknowledgments}

This report was supported by Wonkwang University in 2019. 